\title{
Tipologías para la Innovación tecnológica en Docentes de Educación Superior a partir de un análisis de conglomerados: un estudio exploratorio
}

\section{Typologies for Technological Innovation in Teachers of Higher Education based on an analysis of conglomerates: an exploratory study}

\author{
María Luisa Arancibia Muñoz \\ Universidad Tecnológica de Chile INACAP, Santiago, Chile \\ marancibiam@inacap.cl \\ Ismael Valdivia Zamorano \\ Universidad Tecnológica de Chile INACAP, Santiago, Chile \\ ismael.valdivia@inacapmail.cl \\ Sebastián Mauricio Araneda Riveros \\ Universidad Tecnológica de Chile INACAP, Santiago, Chile \\ seba.araneda.r@gmail.com \\ Julio Cabero-Almenara \\ Universidad de Sevilla, Sevilla, España \\ cabero@us.es
}

\section{Resumen}

En el artículo se analizan las visiones que los docentes tienen respecto a las TIC, y el proceso formativo por el cual pasa para la incorporación de las TIC a su práctica profesional. Concretamente persigue "estudiar si existen diferentes tipologías de docentes respecto a la utilización de las TIC”, y para ello se lleva a cabo una investigación con 370 docentes de todas las carreras y sedes de la Institución de Educación Superior de Chile (INACAP). Para la investigación se construyó un instrumento de recogida de información, elaborado a partir del ECAR Study of Undergraduate Students and Technology y de las dimensiones "utilidad percibida" y "facilidad de utilización" del "Technological Acceptance Model” (TAM). El índice de fiabilidad se obtuvo mediante el coeficiente alfa de Cronbach y alcanzó un valor de 0,927. El análisis estadístico utilizado fue el multivariante de clústers. El resultado del estudio permite diferentes aportaciones, tanto a nivel conceptual, como pragmático e institucional, ya que permite perfilar la toma de decisiones tanto para la integración de las tecnologías, como para establecer planes de formación. Por lo que se refiere a la tipología del docente respecto a las TIC, se han encontrado cuatro grupos, dos con presencia fuerte (la mayoría temprana - 63\%) y mediana presencia (adaptación temprana $30 \%$ ), y dos con una presencia débil (rezagados $-4 \%$ e innovadores $-3 \%$ ). 
Los resultados también avalan los hallazgos encontrados por diferentes estudios.

\title{
Palabras Claves
}

Tecnologías de la Información y Comunicación, formación del profesorado en TIC, uso de las TIC por los docentes, análisis de conglomerados.

\begin{abstract}
The article analyses the visions that teachers have regarding ICT, and the formative process through which they move to the incorporation of ICT in their professional practice. Specifically, it seeks to "investigate whether there are different typologies of teachers regarding the use of ICTs", and for this purpose a research is carried out with 398 teachers from all the careers and headquarters of the Institution of Higher Education of Chile (INACAP). For the research, an instrument for collecting information, based on the ECAR Study of Undergraduate Students and Technology and the "perceived utility" and "ease of use" dimensions of the Technological Acceptance Model (TAM), was constructed. The reliability index was obtained by Cronbach's alpha coefficient and reached a value of 0.927 . The statistical analysis used was the multivariate clusters. The result of the study allows different contributions, at conceptual, pragmatic and institutional level, as it allows to shape, the decision making both for the acquisition of technologies and for establishing training plans. As regards teacher typology in relation to ICT, four groups were found, two with strong (most early - 63\%) and medium presence (early adaptation - 30\%), and two with a weak presence lagging $4 \%$ and innovative - 3\%). The results also support the findings found by different studies.
\end{abstract}

\section{Key Word}

Information and Communication Technologies, teacher training in ICT, use of ICT by teachers, analysis of conglomerates

Tipologías para la Innovación tecnológica en Docentes de Educación Superior a partir de un análisis de conglomerados: un estudio exploratorio. María L. Arancibia, Ismael Valdivia, Sebastián M. Araneda y 


\section{Introducción}

En los últimos años las tecnologías de información y comunicación (TIC), han ido adquiriendo relevancia en los contextos de educación superior. Esto por múltiples motivos, entre ellos por el creciente tránsito hacia modalidades de enseñanza semi-presenciales, denominadas blended-learning (Garrison \& Vaughan, 2008) y en la medida en que estas tecnologías se entienden como herramientas que contribuyen de manera positiva en el proceso de enseñanza y aprendizaje a través de la innovación educativa (Cabero Almenara, 2005; Salinas, 2004). La apropiación de estas tecnologías es especialmente relevante en los docentes, puesto que frecuentemente se menciona que los docentes son una de las influencias más importantes tanto para el uso de las TICs en el proceso de enseñanzaaprendizaje, como para que estas tecnologías sean usadas tanto adentro como afuera del aula por los estudiantes (UNESCO, 2012).

Existen innumerables estudios sobre la importancia de la integración de tecnologías de docentes en general. Por una parte existen estudios que hacen referencia a la integración de elementos específicos como los LMS (Oca, Zermeño, \& Gailbraith, 2015; Rodríguez Espinosa, 2016; Samarawickrema \& Stacey, 2007), otros que abordan el tema desde otros ámbitos como las expectativas, creencias y predisposiciones que pueden tener los docentes de las TICs (Rioseco Pais \& Vila, 2015) y otros solo abordan la caracterización de su uso en general .

Los estudios tienden a identificar ciertos factores claves para la apropiación de la tecnología como son la formación y la alfabetización digital del docente, (Oca et al., 2015; Rangel \& Peñalosa , 2013; Rodríguez , 2016). Otra corriente importante resalta las creencias sobre la enseñanza-aprendizaje de los profesores (Prestridge, 2012; Tondeur, Hermans, van Braak, \& Valcke, 2008) También existen estudios que abordan las características culturales y de funcionamiento del entorno educativo (Samarawickrema \& Stacey, 2007; Tondeur, Devos, Van Houtte, van Braak, \& Valcke, 2009)

Todos estos estudios han logrado identificar ciertas variables que parecieran ser importantes para la integración de las tecnologías, pero sin embargo son muy pocos los estudios que aborden multi-variadamente estos factores (Koh \& Chai, 2014; Tondeur et al., 2008) o que levanten tipologías o clasificaciones de profesores(Martin-García, Hernández \& Sanchez, 2014).

Tipologías para la Innovación tecnológica en Docentes de Educación Superior a partir de un análisis de conglomerados: un estudio exploratorio. María L. Arancibia, Ismael Valdivia, Sebastián M. Araneda y 
Este estudio pretende aportar a la adopción tecnológica por parte de los docentes, tomando como modelos de referencia entre otros a Everett Rogers quien propone una clasificación de adoptantes. Existe un acuerdo general en la literatura de que la adopción implica un tránsito entre un punto inicial hacia un nivel ideal de adopción(González \& Cruz, 2009), Rogers se adscribe a esta consideración al plantear la existencia de niveles: rezagados, mayoría tardía, mayoría temprana, adoptantes tempranos, innovadores (Rogers, 1995). Al construir las clasificaciones de los docentes estaremos en condiciones de identificar variables que pudieran estar incidiendo en que docentes sean más o menos innovadores. Por lo tanto, el presente estudio es explorar las posibles tipologías de adopción tecnológica que presentan los docentes en una Institución de Educación Superior de Chile.

\section{Marco teórico}

Hablar de escenarios formativos en la enseñanza superior en la sociedad del conocimiento, es no olvidarnos que pasan necesariamente por ser entornos que deban ser enriquecidos por las tecnologías, como dirían hace tiempo Dreyer y Nel (2003) por "Technology Enhanced Learning Environment", llamando la atención con ello sobre las posibilidades que las "Tecnologías de la Información y Comunicación" (TIC) estaban incorporando a los procesos formativos, que van desde ampliar los escenarios de formación, facilitar la aplicación de lo aprendido a diferentes contextos, ampliar las posibilidades de interacción de los participantes en la acción formativa, potenciar la creación de entornos de formación multimedia que propicie el desarrollo de diferentes tipos de inteligencias, combinar lo analógico y lo virtual, o deslocalizar la información. Y ello le llevará a que cada vez más los docentes deban ser expertos en una variedad de enfoques basados en la tecnología para la entrega de contenidos, el apoyo al estudiante y la evaluación. (Adams, Cummins, Davis, Freeman, Hall \& Ananthanarayanan, 2017).

Por otra parte, los escenarios actuales tecnológicos se verán fuertemente potenciados en un futuro cercano, con una amplitud de tecnologías, que irán desde la realidad aumentada, las analíticas de aprendizaje, la web semántica, la internet de las cosas, la realidad virtual, (Sharples et al., 2014; Tecnológico de Monterrey, 2015; Johnson, Adams, Cummins, Estrada 2016; Adams, Cummins, Davis, Freeman, Hall \& Ananthanarayanan, 2017). Sin olvidarnos de las posibilidades que empieza aportar la tecnología de la "machine learning" (Mohammed, Badruddin \& Mohammed, 2017).

Ahora bien, su incorporación pasa necesariamente por las actitudes, creencias, formación y grado de aceptación que los docentes tengan hacia ellas (Teo y Noyes, 2011; Tsai y Chai, 2012; Hechter y Vermette, 2013; Teo, Fan, y Du, 2015; Broadbent, 2016; Tondeur, Braak, Ertmer y Ottenbreit-Leftwich, 2016; Ju, Yon \& Hee, 2016; The Scottish

Tipologías para la Innovación tecnológica en Docentes de Educación Superior a partir de un análisis de conglomerados: un estudio exploratorio. María L. Arancibia, Ismael Valdivia, Sebastián M. Araneda y 
Government, 2016; Unesco, 2016; Diep, Zhu, Struyven \& Blieck, 2017; Figueroa, Burgos y Guerrero, 2017).

Por lo que se refiere al modelo de formación utilizado, este ha venido muy marcado por una perspectiva tecnocéntrica, lo que les lleva a tener fuertes competencias tecnológicas-instrumentales para la incorporación de las TIC, pero no tanto de carácter pedagógicas (Prendes y Gutierrez, 2013; Suárez, Almerich, Gargallo y Aliaga, 2013; Cabero-Almenara y Barroso, 2016); de ahí que en los últimos tiempos el modelo formulado Mishra y Koehler (2006) y conocido por las siglas TPACK ("Technological Pedagogical Content Knowledge"), se está proponiendo como uno de los más significativos para ello. El modelo indica que existentes tres fuentes de conocimiento: disciplinar, pedagógico y tecnológico, que en interacción deben establecer las guías que debe seguir los planes de formación y en consecuencia en no centrarse únicamente en los aspectos instrumentales. Aunque revisiones del modelo recientes realizadas del modelo mediante el sistema de ecuaciones estructurales (Ay, Karada y Acat, 2015; Pamuk, Ergun, Cakir, Yilmaz, y Ayas, 2015), han puesto de manifiesto su debilidad para explicar algunas de las interacciones que se dan entre las tres dimensiones del modelo (tecnológica, pedagógica y de contenido). De todas formas, sigue siendo una propuesta válida y útil para la replantear la acción formativa de los docentes (Cejas, Navío y Barroso, 2016; Cabero-Almenara y Barroso, 2016).

Respecto a la difusión y aceptación de la tecnología, una de las propuestas con una fuerte tradición en el ámbito científico es la elaborada por Rogers (1983), conocida como "Teoría de la Difusión de Innovaciones", que en líneas generales lo que viene a señalar que toda innovación o idea, práctica u objeto que es percibida como nueva por la persona o colectivo, no es adoptada directa y completamente, sino que pasa por un proceso antes de su adopción; por tanto, es una teoría que persigue comprender y explicar el proceso seguido para la adopción y la inclusión de las tecnologías en un entramado social. Roger diferencia entre innovación de producto y de proceso, refiriéndose la primera en el terreno educativo por ejemplo a un campus virtual, y la segunda, a una aplicación metodológica del mismo.

Para Roger (1983) el proceso de adopción tecnológica pasa por diferentes etapas: conocimiento (la persona o colectivo toma conocimiento de su existencia), persuasión (el colectivo se forma una opinión favorable o desfavorable respecto a las bondades y posibilidades de la misma), decisión (se adoptan una serie de medidas para adoptar o rechazar el elemento tecnológico), implementación (se incorpora a las actividades usuales realizadas por la persona o el colectivo), y confirmación (se justifica conceptualmente las decisiones adoptadas por los resultados alcanzados). Al mismo tiempo sugiere que en las personas suelen darse diferentes grupos de adopción de las tecnologías en función del momento histórico en la cual la adoptan y que establece en: innovadores (son los primeros que tienden a adoptarlas), primeros seguidores, mayoría precoz, mayoría tardía y rezagados (los últimos en adoptarlas); cada uno de ellos con un porcentaje de penetración (figura1).

Tipologías para la Innovación tecnológica en Docentes de Educación Superior a partir de un análisis de conglomerados: un estudio exploratorio. María L. Arancibia, Ismael Valdivia, Sebastián M. Araneda y 


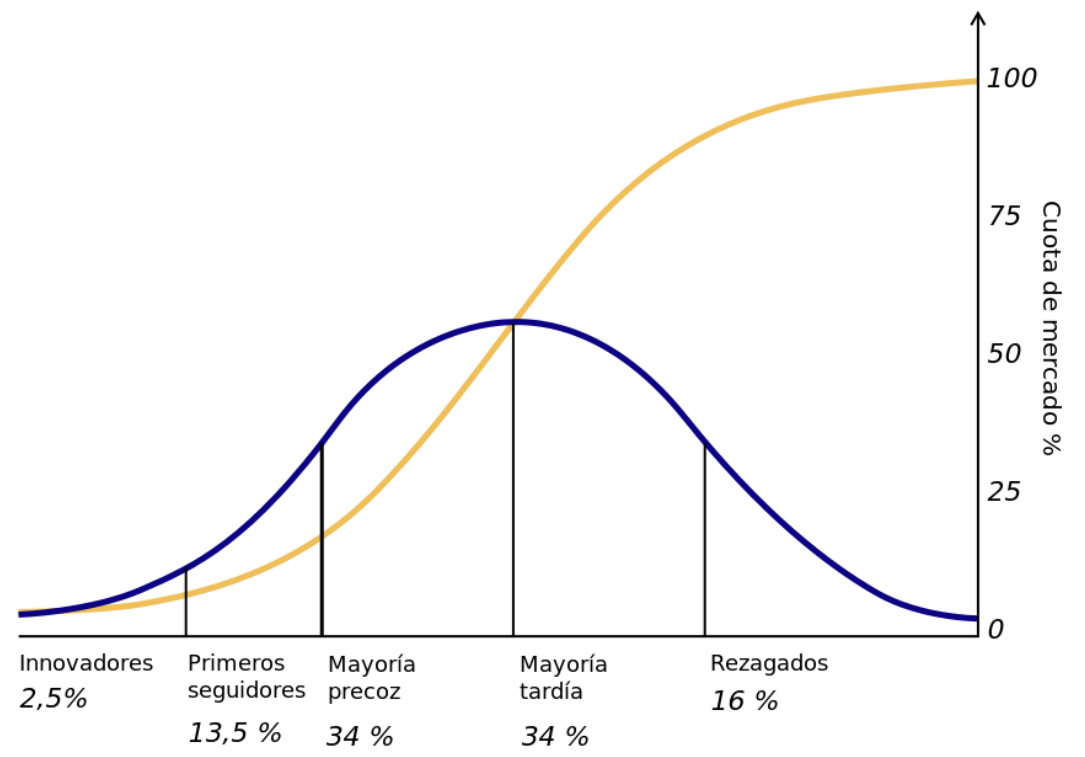

Figura 1 Fases niveles de adopción de la tecnología según Roger (1983)

La propuesta de Roger, aunque formulada en 1969 sigue utilizándose en la actualidad para el análisis de la incorporación de diferentes productos y procesos tecnológicos en distintos contextos y campos: ciencias de la salud (Alonso y Arcila, 2014), sociopolítica tecnológica (Zanello, Fu, Mohnen y Ventresca, 2016; Billon, Lera-Lopez y Marco, 2017), la influencia de la edad (Arpaci, 2015), incorporación de nuevos productos tecnológico (Frattini, Bianchi, De Massis y Sikimic, 2014; Porter y Graham, 2016), o aprendizaje de segundas lenguas Grgurović (2014). También se ha aplicado a la incorporación educativa de las tecnologías (Reyes y Guevara, 2009; Martín-García, Hernández y Sánchez, 2014; Porter y Graham, 2015). Lo que lleva a señalar que es una teoría que sigue teniendo vigencia para explicar el proceso de adopción tecnológica por las personas y las instituciones (Srivastava y Moreland, 2012).

De acuerdo con la teoría se podrían identificar distintos tipos de profesores innovadores, respecto a las TIC, que comprendería diferentes tipos de grupos:

1) Rezagados: Es la categoría de personas más contraria al cambio, se muestran indiferentes ante cualquier innovación e incluso llegan a oponerse a ella y a combatirla activamente; son celosos guardianes del estatus quo y con frecuencia nunca llegan a adoptar la innovación.

2) Mayoría tardía: Son sumamente resistentes a cambiar, son difíciles de persuadir de adoptar una innovación sin una actividad intensa y una influencia significativa. Este nivel estaría representado por aquellos docentes que presentan indicadores de valoración bajos en referencia a las TIC, en cuanto al uso e importancia de las TIC en los procesos de enseñanza aprendizaje.

Tipologías para la Innovación tecnológica en Docentes de Educación Superior a partir de un análisis de conglomerados: un estudio exploratorio. María L. Arancibia, Ismael Valdivia, Sebastián M. Araneda y 
3) Mayoría precoz: Comprende los actores que no están dispuestos a correr riesgos de diversa índole y arriesgar tiempo y otros recursos, tienen cierta resistencia al cambio, analizan y reflexionan cuidadosamente antes de tomar una decisión; sin embargo, son propensos a aceptar la innovación después de una actividad persuasiva relativamente corta. En este grupo, el docente brinda importancia a las tecnologías y su utilización como recurso de aprendizaje, más aún falta incorporar su utilización en el aula, es un docente que tiene las capacidades para hacer uso de la tecnología de forma dirigida.

4) Primeros seguidores: Adoptan la innovación por primera vez, sin realizar análisis de la misma. Pueden ser líderes reconocidos en su colectivo, y por tanto desempeñar un rol para convencer a otras personas en la incorporación de la innovación. Se espera que en este nivel el docente desarrolle una actitud crítica frente a los recursos disponibles analizando oportunidades que ofrece la tecnología en la gestión de información.

5) Innovadores: Son las personas que principalmente corren el riesgo de incorporar, introducir y difundir la innovación. Por lo general, son los propios productores de la innovación, acompañados de un grupo de personas usuarios entusiastas, que pueden ejercer influencia sobre otras personas. En este nivel se espera que el docente esté dispuesto a utilizar las tecnologías en su quehacer académico innovando su aplicación, brindando importancia al uso de las TIC, así como también los recursos dispuestos por la institución. Se espera que el docente comience un proceso que le permitirá mejorar su productividad y eficiencia a través de la interacción con el estudiante, incorporando herramientas de colaboración y retroalimentación tales como portafolios electrónicos.

Referidos a la incorporación de las TIC, distintos autores han propuestos diferentes modelos para explicar las fases de su incorporación a los procesos de enseñanzaaprendizaje, siendo uno de ellos el modelo SAMR (Sustitución, Ampliación, Modificación y Redefinición), formulado por Puentedura (http://www.hippasus.com/rrpweblog/). Para Garcia-Utrera, Figueroa-Rodriguez y Esquivel-Gámez, (2014), la finalidad de este modelo es ayudar a los docentes a evaluar la forma en que están incorporando las tecnologías en sus aulas y de esta manera, conocer qué tipo de usos de la tecnología tienen un mayor o menor efecto sobre el aprendizaje de los estudiantes. El modelo consta de cuatro niveles (Sustitución, Ampliación, Modificación y Redefinición) y dos capas (Mejora y Transformación) que describen el uso de cualquier tecnología. La "sustitución", es el nivel más bajo y únicamente implica un cambio de una tecnología por otra, sin transformaciones metodológicas; la "ampliación", se cambia una tecnología por otra y se le añaden mejoras técnicas que facilitan la realización de tareas, pero tampoco su utilización implica cambios metodológicos; la "modificación", ya envuelve una transformación metodológica que introduce una forma de rediseñar la tecnología; y finalmente la "redefinición", en el cual se crean nuevas actividades y entornos de formación que facilitan su utilización.

Tipologías para la Innovación tecnológica en Docentes de Educación Superior a partir de un análisis de conglomerados: un estudio exploratorio. María L. Arancibia, Ismael Valdivia, Sebastián M. Araneda y 
Otra de las propuestas es la realizada por Krumsvik (2009), que establece que la competencia digital del profesorado está formada por cuatro componentes básicos que se alcanzan de manera sucesiva: las habilidades digitales básicas, la competencia didáctica con las TIC, las estrategias de aprendizaje y la formación o capacitación digital (figura 2).

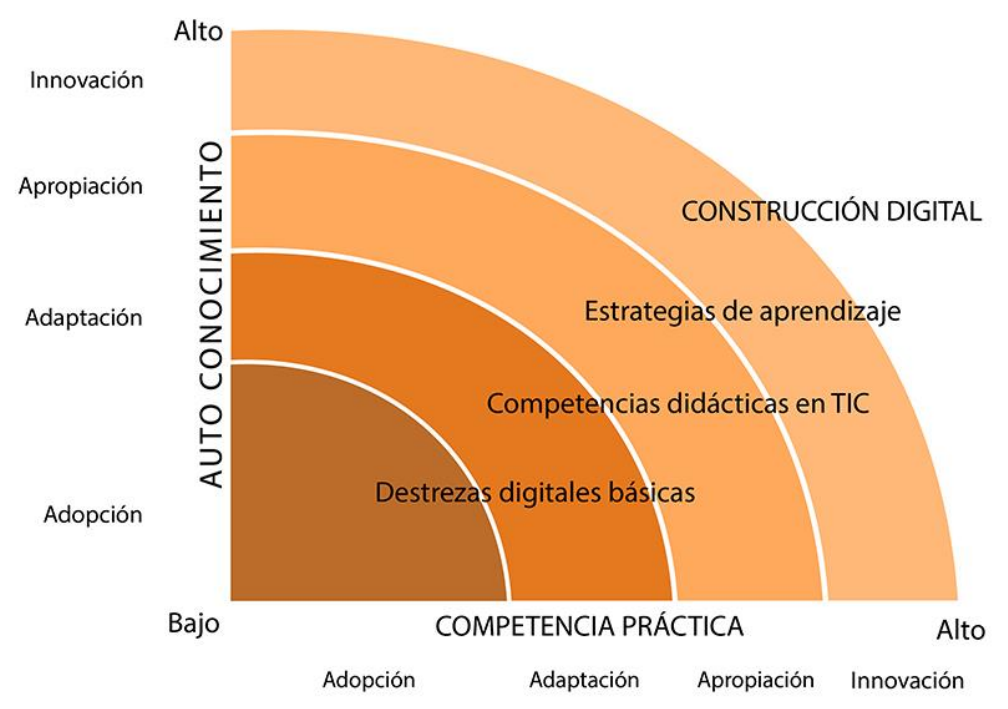

Figura 2. Modelo de adopción de las TIC (Krumsvik, 2009, 178)

El primer nivel comprende las habilidades digitales básicas para el acceso, la gestión, la evaluación, la creación o la comunicación a través de las TIC que debe poseer el docente; en segundo lugar, se encuentra la competencia didáctica con las TIC, que indica que solo tras una correcta mezcla de dominio tecnológico, pedagógico y de contenido se pueden establecer usos correctos de las TIC. El tercer nivel, comprende las estrategias de aprendizaje que moviliza el docente para seguir aprendiendo de forma continua. Y en el último nivel docente adquiere una visión crítica sobre las tecnologías y sus posibilidades.

Siguiendo con el análisis de algunos modelos que revisan el proceso de incorporación de las TIC por los docentes, nos encontramos con las conclusiones que aporta el proyecto ACOT (Apple Classrooms of Tomorrow), proyecto por el cual durante una serie de años la empresa Apple dotó de infraestructura y ayuda tecnológica a diferentes centros de distintos estados norteamericanos (Fisher, 1988; Duyer, 1994; Salas-Castro y Martínez, 2014), con el ánimo de conocer el proceso que los docentes siguen para incorporar las TIC de manera innovadora. Los hallazgos del proyecto indican que en la adopción de una tecnología pasa por diferentes etapas: Acceso (el docente aprende el uso básico de la TIC), adopción (el docente usa la TIC para hacer las mismas cosas que hacia sin ella, y su uso no hay ningún tipo de innovación educativa), adaptación (la TIC es utilizada prácticas tradicionales, pero para aumentar la productividad, el ritmo y la cantidad

Tipologías para la Innovación tecnológica en Docentes de Educación Superior a partir de un análisis de conglomerados: un estudio exploratorio. María L. Arancibia, Ismael Valdivia, Sebastián M. Araneda y 
de información que la comunica al alumno), apropiación (el docente experimenta nuevas maneras de trabajar didácticamente utilizando las TIC, y hacer cosas con ellas que sin ellas no sería posible) e innovación (el docente utiliza la TIC para crear cosas nuevas, que nadie había realizado en su contexto). Indicar al mismo tiempo que el avance a diferentes estadios conlleva tiempo para el docente.

Como estamos observando para la adopción de cualquier TIC, el docente pasa por diferentes fases y etapas, lo que sugiere que dentro de cualquier institución existen docentes con niveles competenciales y los usos diferentes de TIC; así por ejemplo Almerich, Suárez, Orellana, Belloch, Bo y Gastaldo (2005), encuentran cuatro perfiles diferenciados: inicial, inicial-medio, medio y avanzado; señalando que el $72 \%$, de los docentes se sitúan en los dos primeros niveles.

\section{La investigación realizada}

\subsection{Objetivos de la investigación}

El artículo que se presenta forma parte de una investigación más amplia denominada "Conocer la percepción que docentes y estudiantes tenían de la utilidad de las TIC para el aprendizaje y de sus posibilidades para aplicarse a la enseñanza", en el actuar se pretende "estudiar si existen diferentes tipologías de docentes respecto a la utilización de las TIC" en los docentes de educación Superior.

\subsection{Muestra de la investigación}

La muestra de la investigación estuvo formada por 370 docentes, de todas las carreras y sedes de la Institución de Educación Superior de Chile INACAP (http://www.inacap.cl/portal), el nivel de confianza utilizado para determinar el tamaño de la muestra fue un 95\%; la población de docentes es 5234; y en ellas existía representación de profesores de todas las carreras, sedes, géneros y de grupos de mañana y tarde.

\subsection{Instrumentos de recogida de información}

Para la investigación se construyó un instrumento de recogida de información, elaborado a partir del ECAR Study of Undergraduate Students and Technology (Dahlstrom, Walker \& Dziuban, 2013) y de las dimensiones "utilidad percibida" y "facilidad de utilización" del "Technological Acceptance Model” (Davis, 1989).

El instrumento fue sometido al juicio de 5 expertos en investigación educativa, que realizaron recomendaciones en las siguientes direcciones: modificar y mejorar las instrucciones de administración, cambiar redacción de algún ítem para adaptarlo al contexto

Tipologías para la Innovación tecnológica en Docentes de Educación Superior a partir de un análisis de conglomerados: un estudio exploratorio. María L. Arancibia, Ismael Valdivia, Sebastián M. Araneda y 
chileno, agregar algunas opciones de respuestas como "no lo he utilizado", "ambos", recomendaciones que fueron asumidas.

El índice de fiabilidad se obtuvo mediante el coeficiente alfa de Cronbach, y para ello se realizó un estudio piloto con 129 docentes, obteniéndose un valor de 0,927 , que es considerado alto. $\mathrm{Su}$ administración fue vía web: http://www.grupotecnologiaeducativa.es/images/pdf/instrumentos.pdf.

El cuestionario pretendía recoger información de las siguientes grandes dimensiones: rasgos sociodemográficos, uso y propiedad de aparatos tecnológicos, tecnología y experiencia académica, entornos de aprendizaje: presencial y en línea, valoración de la tecnología, ambiente informático personal, utilidad del Moodle y facilidad de uso del Moodle institucional. Salvo en las preguntas de rangos demográficos, en el resto los ítems formulados eran idénticos para los docentes encuestados.

\subsection{Plan de Análisis}

El análisis estadístico utilizado fue el multivariante de clusters (Vallejo, 1992; Everitt, Landau, Leesse y Stahl, 2011) efectuado mediante el programa SPSS (v23).

Por lo que se refiere al "Método Multivariante Clústers" también conocido como análisis de conglomerados, indicar que es una técnica estadística que busca agrupar elementos o variables, tratando de lograr la máxima homogeneidad en cada grupo y la mayor diferencia entre los grupos.

Para su aplicación, y de acuerdo con la propuesta de diferentes autores (Sánchez Carrión, 1984; Vallejo, 1992; Everitt, Landau, Leesse y Stahl, 2011; Vilà-Baños, RubioHurtado, Berlanga-Silvente y Torrado-Fonseca, 2014) se llevó a cabo el siguiente proceso: primero se llevó a cabo un análisis de componentes principales, con el fin de reducir las distintas dimensiones contenidas en el cuestionario. Luego de extraer aquellos factores, se llevó a cabo un análisis de conglomerados a través de la técnica de "k-medias", con el fin de identificar los distintos perfiles o clústers de docentes con respecto a valoración de la tecnología en contextos académicos.

Para el número de factores se consideró a aquellos auto-valores que fueran superiores a 1, ya que esto es lo mínimo que nos garantiza que aquel factor esté explicando al menos la variabilidad de un factor. Esto nos condujo a consolidar 5 factores o componentes que agrupan a cada Clúster. Los valores de cada clúster están en una escala de valoración positiva, con valores posibles son 1 a 5 (no es importante a muy importante), en la tabla 1 se observa el valor promedio (centroide) de cada componente en el clúster.

Los factores de los clúster se basaron en el cuestionario y son los siguientes:

Tipologías para la Innovación tecnológica en Docentes de Educación Superior a partir de un análisis de conglomerados: un estudio exploratorio. María L. Arancibia, Ismael Valdivia, Sebastián M. Araneda y 
1.- Importancia de las TIC: Variables asociadas a la importancia de cada aparato tecnológico para el aprendizaje de los estudiantes

2.-Valoración del Moodle institucional: ítems que se aglomeraron en función de las variables sobre utilidad y facilidad de uso

3.- Interés en los recursos académicos: Ítems que se aglomeraron en función de variables que miden valoración en el ámbito pedagógico de recursos como: Sitio de la biblioteca de la universidad, Herramientas web para bibliografía (EndNote, CiteULike, OttoBib y otros), Sitio Web de la universidad, E-portafolios, Libros y textos digitales, simulaciones o juegos educativos.

4.- Uso de TIC en clases: ítems que se aglomeraron sobre variables que miden uso de la tecnología en la experiencia académica en tres ejes, eficiencia, pertinencia de la tecnología y retroalimentación del docente.

5.- El último componente es la propiedad de la tecnología que posee el docente incluyendo propósitos educativos y generales.

A continuación se observa la conformación de 4 clúster según escala de valoración de 1 a 5 (no es importante a muy importante).

\begin{tabular}{|c|c|c|c|c|}
\hline Componente & Rezagado & $\begin{array}{c}\text { La Clúster } \\
\text { mayoría } \\
\text { temprana }\end{array}$ & $\begin{array}{c}\text { Adoptantes } \\
\text { tempranos }\end{array}$ & Innovador \\
\hline Importancia TICs & 3,2 & 3,7 & 4 & 4,8 \\
\hline Importancia Moodle & 2,3 & 4,3 & 4,3 & 4,3 \\
\hline $\begin{array}{c}\text { Interés por los recursos } \\
\text { Académicos }\end{array}$ & 4,0 & 4,3 & 4,3 & 4,5 \\
\hline $\begin{array}{c}\text { Uso TIC en clases } \\
\text { Propiedad aparatos }\end{array}$ & 3,0 & 3,0 & 4,0 & 4,0 \\
\hline tecnológicos & 2,3 & 3 & 2,3 & 4,3 \\
\hline
\end{tabular}

Tabla 1. Puntuaciones medias o centroides del clúster. Elaboración propia

Luego de aplicado el modelo estadístico, y elegidos los conglomerados, se pueden visualizar ya los grupos conformados, que son homogéneos en su interior y heterogéneos entre sí. Se presentan algunas descripciones de cada agrupación de acuerdo al Modelo de Rogers principalmente.

Tipologías para la Innovación tecnológica en Docentes de Educación Superior a partir de un análisis de conglomerados: un estudio exploratorio. María L. Arancibia, Ismael Valdivia, Sebastián M. Araneda y 
1.- Rezagado y Mayoría tardía : Es el profesor que se resiste al cambio; si bien en el presente análisis se vislumbra que tiene un marcado interés por los recursos académicos, es un profesor que le otorga muy poca importancia a las TICS tanto dentro como fuera del aula y que presenta una muy baja propiedad de aparatos tecnológicos. Es un profesor que le da muy poca importancia al Moodle.

2.- La mayoría temprana o precoz: Es el profesor que tiene mucho interés por los recursos académicos, que le da mucha importancia a las TIC y los utiliza para el aprendizaje, y que por lo tanto también le otorga mucha importancia al Moodle, pero que aún le falta incorporar su utilización en el aula. Es un docente que por lo demás no tiene mucha propiedad de aparatos tecnológicos, lo que le puede implicar no poseer un manejo tecnológico óptimo. Son propensos a aceptar la innovación, lo que los podría diferenciar de los rezagados, puesto que estos mantienen una actitud abierta a ella.

3.- Adoptador temprano o primeros seguidores: Es un docente que le otorga mucha importancia a las TIC y que hace mucho énfasis por el uso de TICS en clases. Lo único que lo distancia del innovador es la propiedad de aparatos tecnológicos, lo que puede implicar una menor apropiación de estas herramientas. Los adoptadores tempranos son quienes adoptan la innovación por primera vez, sin mucha discusión y análisis; pueden actuar como líderes reconocidos y respetados y jugar un papel importante para persuadir a otros actores de adoptar la innovación.

4.- Innovador: El docente innovador le otorga mucha importancia a las TIC. Presenta también mucho interés en los recursos académicos y lo que los diferencia de los otros es que además de estas valoraciones, es que además presenta una alta propiedad de aparatos tecnológicos. Probablemente la propiedad de aparatos tecnológicos, puede tener algo que ver con la apropiación de las herramientas tecnológicas. En este nivel se espera que el docente reconozca en la plataforma Moodle, un aliado estratégico para sus tareas de gestión de información, son aquellos docentes que pueden liderar procesos de capacitación y promoción del uso de las TIC.

En la tabla 2, se presenta cómo se distribuyeron los docentes de la universidad investigada, en relación a las tipologías de profesores que el modelo de conglomerados propone.

\begin{tabular}{|c|c|c|c|}
\hline Clúster & $\begin{array}{c}\text { Clasificación en base a } \\
\text { Rogers }\end{array}$ & Frecuencia & Proporción \\
\hline $\mathbf{1}$ & Rezagado & 14 & $4 \%$ \\
\hline $\mathbf{2}$ & La mayoría temprana & 232 & $63 \%$ \\
\hline $\mathbf{3}$ & Adoptador temprano & 112 & $30 \%$ \\
\hline $\mathbf{4}$ & Innovador & 12 & $3 \%$ \\
\hline & Total & 370 & $100 \%$ \\
\hline
\end{tabular}

Tipologías para la Innovación tecnológica en Docentes de Educación Superior a partir de un análisis de conglomerados: un estudio exploratorio. María L. Arancibia, Ismael Valdivia, Sebastián M. Araneda y 
Tabla 2. Clúster en base a variables de interés. Elaboración propia

En la tabla 2 se presentan las proporciones de los grupos definidos en los docentes. Se puede apreciar que la tipología de docente que más preponderancia tiene en la muestra de docentes encuestados es la de "mayoría temprana" que corresponde a un $63 \%$. Es relevante hacer énfasis en este dato, ya que nos podría estar dando indicios de donde se sitúa la mayoría de los profesores. Este docente es uno que le otorga una importancia considerable a la tecnología, pero que probablemente en la práctica le dificulte, puesto que presentan baja propiedad de aparatos tecnológicos, y bajo uso de TIC en clases. Es decir, no sería un problema de actitud hacia la tecnología su utilización, sino posiblemente por falta de formación. Se puede también destacar el grado de semejanza de la gráfica alcanzada con la representación gráfica (figura 1) de las fases niveles de adopción de la tecnología según Roger (1983).

La segunda mayoría tiene que ver con el docente que es el adoptador temprano (30\%), lo que nos dice que una importante proporción de la muestra de docentes encuestados, están muy cercanos a ser docentes innovadores.

Finalmente señalar que los grupos "rezagados" (4\%) e "innovador" (3\%), tienen baja presencia en los docentes, y con porcentajes muy similares.

A continuación se presentan los clúster en una representación gráfica lo que permite visualizar los resultados obtenidos en los diferentes componentes. Es posible apreciar en la Figura 4 el grupo de docentes denominados "innovador" (línea segmentada) se encuentran valoraciones altas en todos los componentes considerados a diferencia del segundo grupo denominado "adoptantes tempranos" (línea doble) son aquellos que presentan puntuaciones altas en los componentes de importancia de Tics y Moodle, interés por recursos académicos y que hacen uso de las TIC, tienen puntuación baja en propiedad de aparatos tecnológicos. El tercer grupo denominado "mayoría temprana" (línea punteada) presenta valoraciones altas relacionadas con la importancia del Moodle e interés por utilizarlo, pero en el uso de la tecnología tiene un valor menor $(3,0)$. Y el último grupo del "Rezagado" presenta resultados bajos en 4 de los 5 componentes, sólo presenta interés en los recursos académicos.

Tipologías para la Innovación tecnológica en Docentes de Educación Superior a partir de un análisis de conglomerados: un estudio exploratorio. María L. Arancibia, Ismael Valdivia, Sebastián M. Araneda y 


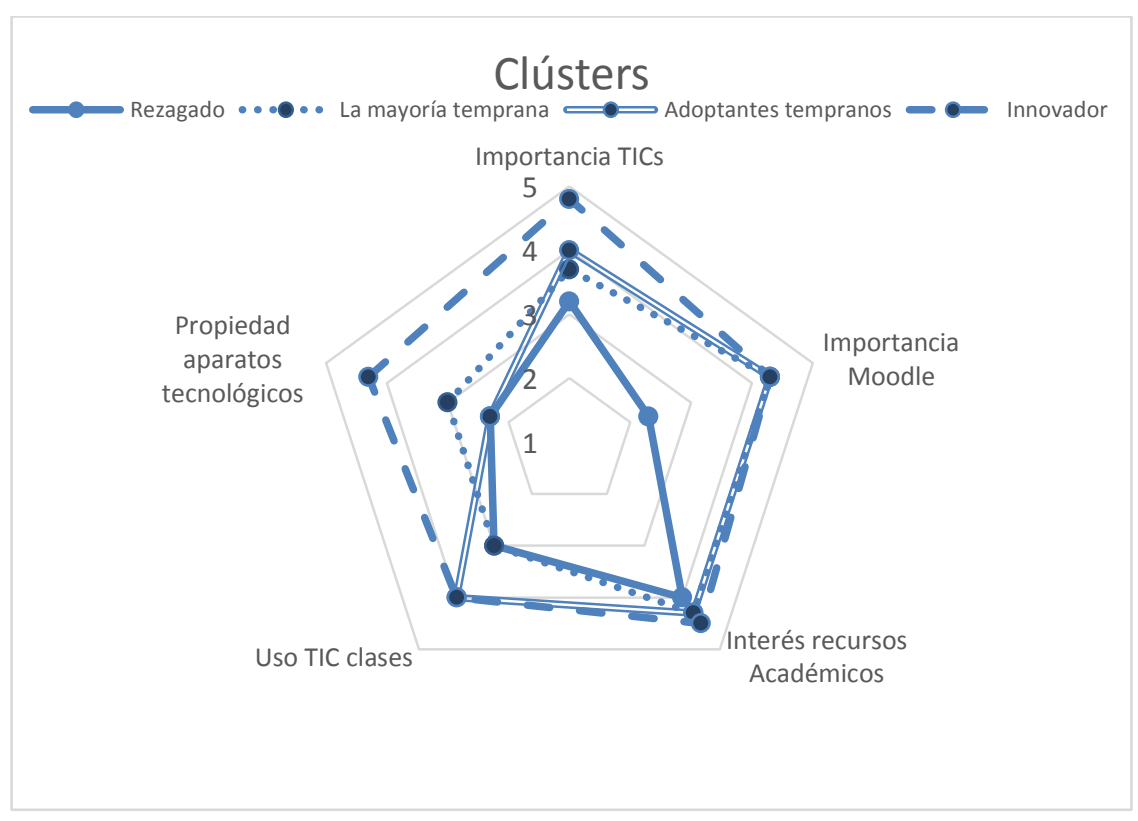

Figura 3. Representación de los clúster

\section{Conclusiones e implicaciones}

Estudios de este tipo permite indicar diferentes aportaciones, tanto a nivel conceptual, pragmático e institucional, ya que permite perfilar la toma de decisiones tanto para la adquisición de tecnologías, como para establecer planes de formación. El estudio por tanto es relevante en dimensiones pedagógicas e institucionales. En sus aspectos pedagógicos porque ofrece lineamientos con respecto a qué factores individuales y sociales intervienen en la adopción y la innovación en la educación; y además otorga conocimiento con una fuerte base empírica respecto a la proporción de docentes que pertenece a cada tipología, con el fin de tener una fotografía del comportamiento del cuerpo docente en general de una institución con respecto a la tecnología. Esto último puede ser bastante útil desde el punto de vista institucional en el sentido de que otorga referencias sólidas, para abordar de manera más eficiente e informada estrategias de promoción de la tecnología, la innovación educativa y la formación del profesorado.

Por lo que se refiere a la tipología del docente respecto a las TIC, se han encontrado cuatro grupos, dos con fuerte presencia que son: (la mayoría temprana - 63\% y (adaptación temprana -30\%), y dos con una presencia débil (rezagados $4 \%$ e innovadores 3\%). Ello ya nos indica como por ejemplo sugiere el modelo formulado por Krumsvik (2009) y el modelo ACOT (Fisher, 1988; Duyer, 1994; Salas-Castro y Martínez, 2014), por una parte, que no hay un único estadio, sino que se encuentra una diversidad de ellos, y por otra, que los docentes de esta institución se encontrarían de adaptación y adopción. Por como se desprende de los datos obtenido en nuestro estudio, y que coincide con los trabajos

Tipologías para la Innovación tecnológica en Docentes de Educación Superior a partir de un análisis de conglomerados: un estudio exploratorio. María L. Arancibia, Ismael Valdivia, Sebastián M. Araneda y 
realizados sobre los modelos SAMR y ACOT (Fisher, 1988; Duyer, 1994; Salas-Castro y Martínez, 2014), existe una diversidad de niveles de dominio tecnológico por parte de los docentes, de ahí la necesidad de establecer en los centros planes de formación a distintos niveles y profundidades.

Los resultados también avalan los hallazgos encontrados por diferentes estudios (Teo y Noyes, 2011; Tsai y Chai, 2012; Hechter. y Vermette, 2013; Teo, Fan, y Du, 2015; Broadbent, 2016; Tondeur, Braak, Ertmer y Ottenbreit-Leftwich, 2016; Ju, Yon \& Hee, 2016; The Scottish Government, 2016; Van den Beemt y Diepstraten, 2016; Diep, Zhu, Struyven \& Blieck, 2017), que indican que los docentes suelen tener actitudes positivas para incorporar las TIC, pues como se desprende del estudio la gran mayoría de docentes se sitúan en el terreno de la adopción de las tecnologías Al mismo tiempo el fuerte porcentaje de docentes situados en el clúster de "la mayoría avanzada", nos sugiere que la formación que se realice, y siguiendo el modelo del TPACK, deberá centrarse principalmente en las dimensiones didácticas y disciplinar (Mishra y Koehler, 2006), y no exclusivamente técnica, como suele realizarse.

Llevando a cabo un análisis más profundo, señalar en primer lugar, y como ya se hizo mención, el estudio revela la existencia de profesores que en su mayoría valoran positivamente la tecnología, pero que, en una importante proporción, no la usa. Sería relevante indagar más en profundidad cuales son las limitantes de uso que tienen los profesores, puesto que en el modelo del clúster, aparece como una diferencia significativa entre un profesor de "mayoría precoz", a "primeros seguidores", que son las categorías donde se aglomeran la mayor proporción de docentes.

En segundo lugar, el trabajo revela la importancia de la propiedad de aparatos tecnológicos, como un factor que distancia al profesor de "mayoría precoz" del "innovador". La conjetura es que probablemente la propiedad de aparatos tecnológicos puede tener alguna relación con la facilidad de uso de las distintas herramientas. Sería interesante explorar esta relación, puesto que podría ser sustantivamente relevante para la adopción de la tecnología. Si se comprobara esta relación, sería coherente planear estrategias en la dirección de incentivar la propiedad de aparatos tecnológicos. De todas formas esta conclusión debe tomarse con cautela, pues se puede caer en el error de pensar que la simple incorporación de TIC puede servir para que un profesor sea innovador con ellas. Más bien debemos entenderlo, como que un docente que adquiere una tecnología presenta ya un cierto grado de aceptación de la misma, y como sugiere el modelo TAM de adopción de las tecnologías formulado por Davis (1989), el grado de aceptación repercute sobre la intención de su utilización.

En tercer lugar, podemos señalar que existencia de una mínima proporción de profesores pertenecientes a la tipología "rezagados", es natural en cualquier institución que propicia la innovación pedagógica con apoyo de tecnología, es un profesor que a pesar de demostrar interés en recursos académicos, presenta un bajo interés por las TIC y su uso en clases, y un bajo interés en el Moodle que es la herramienta más valorada por la

Tipologías para la Innovación tecnológica en Docentes de Educación Superior a partir de un análisis de conglomerados: un estudio exploratorio. María L. Arancibia, Ismael Valdivia, Sebastián M. Araneda y 
generalidad de los profesores. Sería significativo para el aspecto tecnológico de la institución indagar en cuáles son las razones de estas resistencias, y bajas valoraciones. Esta situación nos lleva a proponer, la necesidad de que desde la institución se establezcan planes de capacitación en TIC para este colectivo, que desde nuestro punto de vista debe tener un fuerte componente actitudinal para que los docentes cambien las percepciones iniciales negativas que pueden tener hacia las TIC.

Y en cuarto lugar, podría resultar relevante la proporción de profesores "mayoría temprana" $(30 \%)$ puesto que están muy cercanos a ser innovadores. Una estrategia específica para potenciar a estos profesores, sería relevante en el sentido de perfilarlos como actores relevantes en la transferencia del conocimiento sobre el uso de las herramientas entre sus pares.

Presentación del artículo: 12 de octubre de 2017 Fecha de aprobación: 16 de noviembre de 2017 Fecha de publicación: 22 de diciembre de 2017

Arancibia, M.L, Valdivia, I., Araneda, S.M, y Cabero-Almenara, J. (2017). Tipologías para la Innovación tecnológica en Docentes de Educación Superior a partir de un análisis de conglomerados: un estudio exploratorio. RED. Revista de Educación a Distancia, 55. Recuperado de: http://www.um.es/ead/red/55/arancibia_et_al.pdf

\section{Financiación}

El presente trabajo se inserta en el proyecto de investigación denominado "Conocer la percepción que docentes y estudiantes tenían de la utilidad de las TIC para el aprendizaje y de sus posibilidades para aplicarse a la enseñanza “, financiado por el Centro de Innovación de Educación CIEDU de la Universidad Tecnológica de Chile, INACAP - Chile

\section{5.- Referencias bibliográficas}

Adams, S., Cummins, M., Davis, A., Freeman, A., Hall, C., \& Ananthanarayanan, V. (2017). NMC Horizonte Report: 2017 Higher Education Edition. Austin, Texas: The New Media Consortium.

Almerich, G., Suárez, J.M., Orellana, N., Belloch, C., Bo, R. \& Gastaldo, I. (2005). Diferencias en los conocimientos de los recursos tecnológicos en profesores a partir del género, edad y tipo de centro. RELIEVE, 11(2), 127-146.

Alonso, L. \& Arcila, C. (2014). La teoría de Difusión de Innovaciones y su relevancia en la promoción de la salud y prevención de la enfermedad. Salud Uninorte, 30(3), 451-464.

Tipologías para la Innovación tecnológica en Docentes de Educación Superior a partir de un análisis de conglomerados: un estudio exploratorio. María L. Arancibia, Ismael Valdivia, Sebastián M. Araneda y 
Arpaci, I. (2015). A comparative study of the effects of cultural differences on the adoption of mobile learning. British Journal of Educational Technology. 46(4), 699-712. doi:10.1111/bjet.12160.

Ay, Y., Karada, E. \& Acat, M. (2015). The Technological Pedagogical Content Knowledge-practical (TPACK Practical) model: Examination of its validity in the Turkish culture via structural equation modeling. Computers \& Education, 88, 97-108. http://dx.doi.org/10.1016/j.compedu.2015.04.017.

Billon, M. \& Lera-Lopez, F. y Marco, R. (2017). Patterns of Combined ICT Use and Innovation in the European Regions. Journal of Global Information Technology Management, 20(1), 28-42, DOI: 10.1080/1097198X.2017.1280302.

Broadbent, J. (2016). Academic success is about self-efficacy rather than frequency of use of the learning management system. Australasian Journal of Educational Technology, 32(4), 38-49.

Cabero-Almenara, J. \& Barroso, J. (2016) ICT Teacher training: a view of the TPACK model / Formación del profesorado en TIC: una visión del modelo TPACK. Cultura y Educación, 28(3), 633-663, doi: http://dx.doi.org/10.1080/11356405.2016.1203526.

Cabero-Almenara, J. (2005). Las TICs y las Universidades: retos, posibilidades y preocupaciones. Revista de La Educación Superior, XXXIV (3), 77-100. Retrieved from http://goo.gl/YzNzaW

Cejas, R. Navío, A. \& Barroso, J. (2016). Las competencias del profesorado universitario desde el modelo TPACK (conocimiento tecnológico y pedagógico del contenido). Pixel-Bit. Revista de Medios y Educación, 49, 105-119. doi: http://dx.doi.org/10.12795/pixelbit.2016.i49.07.

Dahlstrom, E., Walker, J. D., \& Dziuban, C. (2013). ECAR Study of Undergraduate Students and Technology, Educause Centre for Applied Research, (February), 1-12.

Davis, F. D. (1989). Perceived Usefulness, Perceived Ease of Use, and User Acceptance of Information Technology. (M. I. Center, Ed.)

Diep, A., Zhu, Ch., Struyven, K \& Blieck, Y. (2017). Who or what contributes to student satisfaction in different blended learning modalities? British Journal of Educational Technology, 48(2), 473-489. doi:10.1111/bjet.12431.

Dreyer, C. \& Nel, Ch. (2003). Teaching reading strategies and reading comprehension within a technology-enhanced learning environment. System, 31(3), 349-365. https://doi.org/10.1016/S0346-251X(03)00047-2.

Duyer, D. (1994). Apple Classrooms of Tomorrow: What We've Learned. Educational Leadirship, 51(5), 4-10.

Everitt, B., Landau, S., Leesse, M. \& Stahl, D. (2011). Cluster Analysis. Hoboken: Wiley.

Figueroa, V., Burgos, F. \& Guerrero, M. (2017). Actitud de los docentes hacia el uso de la

Tipologías para la Innovación tecnológica en Docentes de Educación Superior a partir de un análisis de conglomerados: un estudio exploratorio. María L. Arancibia, Ismael Valdivia, Sebastián M. Araneda y 
computadora en las escuelas de República Dominicana. Pixel-Bit. Revista de Medios y Educación, 51, 197-210. Doi: http://dx.doi.org/10.12795/pixelbit.2017.i51.13.

Fisher, CH. (1988). The influence of High Computer Access on Schoolwork and Student Empowerment: An Exploratory Study of the Nashville ACOT Site. Apple Computer, Inc., Cupertino, CA.

Frattini, F., Bianchi, M., De Massis, A. \& Sikimic, U. (2014). The Role of Early Adopters in the Diffusion of New Products: Differences between Platform and Nonplatform Innovations. Journal of Prouct Innovation Management, 31(3), 466-488. DOI: 10.1111/jpim.12108.

Garcia-Utrera, L., Figueroa-Rodríguez, S. \& Esquivel-Gámez, I. (2014). Modelo de Sustitución, Aumento, Modificación, y Redefinición (SAMR): Fundamentos y aplicaciones. En Esquivel-Gámez (Coord.), Los Modelos Tecno-Educativos: Revolucionando el aprendizaje del siglo XXI (pp. 205-220). México: DSAEUniversidad Veracruzana.

Garrison, D. R., \& Vaughan, N. D. (2008). Blended learning in higher education: Framework, principles, and guidelines. booksgooglecom (Vol. 1st). Retrieved from http://www.amazon.com/dp/0787987700

González, D., \& Cruz, H. (2009). Adopción de las Tecnologías Infocomunicacionales (TI) en docentes: actualizando enfoques. Revista Electrónica Teoría de La Educación, 10, 134-150. Retrieved from http://dialnet.unirioja.es/

Grgurović, M. (2014). An application of the Diffusion of Innovations theory to the investigation of blended language learning, Innovation in Language Learning and Teaching, 8(2), 155-170, DOI: 10.1080/17501229.2013.789031.

Hechter, R.P. \& Vermette, L.A. (2013). Technology integration in K-12 science classrooms: An analysis of barriers and implications. Themes in Science \& Technology Education, 6(2), 73-90.

Salinas. J. (2004). Innovación docente y uso de las TIC en la enseñanza universitaria. Revista Universidad y Sociedad Del Conocimiento, 1(1), 16. https://doi.org/10.1007/s13398-014-0173-7.2

Johnson, L. Adams, S., Cummins, M., Estrada, V., Freeman, A. \& Hall, C. (2016). NMC Horizon Report: 2016 Higher Education Edition. Austin, Texas: The New Media Consortium.

Ju, Y., Yon, K. \& Hee, N. (2016). The effects of secondary teachers' technostress on the intention to use technology in South Korea. Computers \& Education, 95, 114-122. Doi: http://dx.doi.org/10.1016/j.compedu.2015.12.004.

Koh, J. H. L., \& Chai, C. S. (2014). Teacher clusters and their perceptions of technological pedagogical content knowledge (TPACK) development through ICT lesson design. $\begin{array}{llll}\text { Computers and 222-232. } & \text { Education, }\end{array}$

Tipologías para la Innovación tecnológica en Docentes de Educación Superior a partir de un análisis de conglomerados: un estudio exploratorio. María L. Arancibia, Ismael Valdivia, Sebastián M. Araneda y 
https://doi.org/10.1016/j.compedu.2013.08.017

Krumsvik, R. (2009). Situated learning in the network society and the digitised school. European Journal of Teacher Education, 32(2), 167-185, DOI: $10.1080 / 02619760802457224$.

Martín-García, A., Hernández, M.J. \& Sánchez, M.C. (2014). Fases y clasificación de adoptantes de blended learning en contextos universitarios. Aplicación del análisis CHAID. Revista Española de Pedagogía, 259, 457-476.

Mishra, P., \& Koehler, M. (2006). Technological pedagogical content knowledge: A framework for teacher knowledge. The Teachers College Record, 108(6), 1017-1054.

Mohammed, M., Badruddin, M. y Mohammed, B. (2017). Machine learning: algorithms and applications. Florida: CRC.

Oca, Á. R. M. M. de, Zermeño, M. G. G., \& Gailbraith, L. A. G. (2015). Uso de la plataforma Moodle como apoyo a la docencia presencial universitaria. EDMETIC, 4(1), 135-155.

Pamuk, S., Ergun, M., Cakir, R., Yilmaz, y Ayas, C. (2015). Exploring relationships among TPACK components and development of the TPACK instrument. Educational Informational Technology, 20, 241-263. Doi 10.1007/s10639-013-9278-4.

Porter, W. y Graham, Ch. (2015). Institutional drivers and barriers to faculty adoption of blended learning in higher education. British Journal of Educational Technology, 47(4), 748-762. doi:10.1111/bjet.12269.

Prendes, M.P. \& Gutiérrez, I. (2013). Competencias tecnológicas del profesorado en las universidades españolas. Revista de Educación, 361, 196-222.

Prestridge, S. (2012). The beliefs behind the teacher that influences their ICT practices. Computers and Education, 58(1), 449-458. https://doi.org/10.1016/j.compedu.2011.08.028

Rangel Baca, A., \& Peñalosa Castro, E. A. (2013). Alfabetización digital en docentes de educación superior: construcción y prueba empírica de un instrumento de evaluación. Pixel-Bit. Revista de medios y educación, (43), 9-23. https://doi.org/10.12795/pixelbit.2013.i43.01

Reyes, D. \& Guevara, H. (2009). Adopción de las tecnologías Infocomunicacionales (TIi) en docentes: actualizando enfoques. Revista Electrónica Teoría de la Educación. Educación y Cultura en la Sociedad de la Información. 10(1), 134-150.

Rioseco Pais, M. H., \& Vila, R. (2015). Expectativas que poseen los docentes universitarios de carreras de Pedagogía en relación al uso de las TIC. Pixel-Pixel. Revista de Medios y Educacion, (46), 51-64.

Rodríguez Espinosa, H. (2016). Desarrollo de habilidades digitales docentes para implementar ambientes virtuales de aprendizaje en la docencia universitaria. Sophia,

Tipologías para la Innovación tecnológica en Docentes de Educación Superior a partir de un análisis de conglomerados: un estudio exploratorio. María L. Arancibia, Ismael Valdivia, Sebastián M. Araneda y 
12(2), 261. https://doi.org/10.18634/sophiaj.12v.2i.561

Rogers, E. M. (1983). Diffusion of innovations. New York. Free Press.

Rogers, E. M. (1995). Diffusion of innovations. Macmillian Publishing Co. https://doi.org/citeulike-article-id:126680

Salas-Castro, R. \& Martínez, J. (2014). Aulas Apple del mañana: Resultados empíricos de educación básica (Modelo ACOT). En Esquivel-Gámez (Coord.), Los Modelos TecnoEducativos: Revolucionando el aprendizaje del siglo XXI (pp. 17-32). México: DSAEUniversidad Veracruzana.

Samarawickrema, G., \& Stacey, E. (2007). Adopting Web-Based Learning and Teaching: A case study in higher education. Distance Education, 28(3), 313-333. Doi: https://doi.org/10.1080/01587910701611344

Sharples, A. et al (2014). Innovating Pedagogy 2014. Exploring new forms of teaching, learning and assessment, to guide educators and policy makers. Milton Keynes: The Open University.

Srivastava, J. \& Moreland, J. (2012). Diffusion of Innovations: Communication Evolution and Influences. The Communication Review, 15(4), 294-312, DOI: 10.1080/10714421.2012.728420.

Suárez, J., Almerich, G. Gargallo, B. \& Aliaga, F. (2013). Las competencias del profesorado en TIC: estructura básica. Educación XXI, 16, 1, 39-62. DOI: 10.5944/educxx1.16.1.716.

Tecnológico de Monterrey (2015). Reporte EduTrends. Radar de Innovación Educativa 2015. Monterrey: Tecnológico de Monterrey.

Teo, T. \& Noyes, J. (2011). An assessment of the influence of perceived enjoyment and attitude on the intention to use technology among pre-service teachers: a structural equation modeling approach. Computers \& Education, 57(2), 1645-1653.

Teo, Th. Fan, X. \& Du, J. (2015). Technology acceptance among pre-service teachers: Does gender matter? Australasian Journal of Educational Technology, 31(3), 235251.

The Scottish Government (2016). Enhancing learning and teaching through the use of digital technology. Edinburgh: The Scottish Government.

Tondeur, J., Braak, J., Ertmer, P., \& Ottenbreit-Leftwich, A. (2016). Understanding the relationship between teachers' pedagogical beliefs and technology use in education: a systematic review of qualitative evidence. Education Tech Research Dev. DOI 10.1007/s11423-016-9481-2.

Tondeur, J., Devos, G., Van Houtte, M., van Braak, J., \& Valcke, M. (2009). Understanding structural and cultural school characteristics in relation to educational change: the case of ICT integration. Educational Studies, 35(2), 223-235.

Tipologías para la Innovación tecnológica en Docentes de Educación Superior a partir de un análisis de conglomerados: un estudio exploratorio. María L. Arancibia, Ismael Valdivia, Sebastián M. Araneda y 
https://doi.org/10.1080/03055690902804349

Tondeur, J., Hermans, R., van Braak, J., \& Valcke, M. (2008). Exploring the link between teachers' educational belief profiles and different types of computer use in the classroom. Computers in Human Behavior, 24(6), 2541-2553. https://doi.org/10.1016/j.chb.2008.02.020

Tsai, C.-C. \& Chai, C. S. (2012). The "third"-order barrier for technology integration instruction: Implications for teacher education. Building the ICT capacity of the next generation of teachers in Asia. Australasian Journal of Educational Technology, 28(6), 1057-1060.

Unesco (2016). Tecnologías digitales al servicio de la calidad educativa. Unesco: Santiago de Chile.

Unesco (2012). ICT in education in Latin America and the Caribbean a regional analysis of ICT integration and e-readiness. Unesco: Montreal.

Vallejo, G. (1992). Técnicas multivariantes aplicadas a las ciencias del comportamiento. Oviedo: Servicio de Publicaciones de la Universidad de Oviedo.

Van den Beemt, A. y Diepstraten, I. (2016). Teacher perspectives on ICT: A learning ecology approach. Computers \& Education, 92-93, 161-170.

Vilà-Baños, R. Rubio-Hurtado, M. J., Berlanga-Silvente, V., \& Torrado-Fonseca, M. (2014). Cómo aplicar un clúster jerárquico en SPSS. REIRE, Revista d'Innovació $i$ Recerca en Educació, 7(1), 113- 127.

Zanello, G., Fu, X., Mohnen, P. \& Ventresca, M. (2016). The creation and diffusion of innovation in developing countries: a systematic literature review. Journal of Economic Surveys. 30(5), 884-912. Doi: 10.1111/joes.12126.

Tipologías para la Innovación tecnológica en Docentes de Educación Superior a partir de un análisis de conglomerados: un estudio exploratorio. María L. Arancibia, Ismael Valdivia, Sebastián M. Araneda y 